\title{
Empresa familiar: La importancia del clima familiar y la identificación de la familia con su empresa
}

\author{
María Katiuska Cabrera-Suárez ${ }^{1}$, María de la Cruz Déniz-Déniz, Josefa Delia Martín-Santana \\ Universidad de Las Palmas de Gran Canaria, España \\ doi: 10.20420 /eni.2020.326
}

\begin{abstract}
Resumen
Este trabajo analiza cómo el clima familiar afecta el establecimiento de objetivos no financieros o socioemocionales en las empresas familiares, y sugiere que esta influencia está mediada por el grado de identificación de la familia con la empresa. Más específicamente, proponemos que dinámicas familiares positivas generan identificación de la familia con su empresa. A su vez, la identificación lleva a la empresa a adoptar objetivos no financieros. Estas hipótesis se ven confirmadas por los datos obtenidos de 374 miembros de equipos de alta dirección en 173 empresas familiares no cotizadas españolas.
\end{abstract}

Palabras clave: empresa familiar, clima familiar, identificación, objetivos no financieros, EADs, riqueza socioemocional.

Clasificación JEL: M10, M14.

Agradecimiento: Las autoras agradecen el apoyo financiero proporcionado por el Ministerio de Ciencia e Innovación al Proyecto ECO2008-00265/ ECON.

Fuente de referencia: Cabrera-Suárez, M. K., Déniz-Déniz, M. D. L. C., \& Martín-Santana, J. D. (2014). The setting of nonfinancial goals in the family firm: The influence of family climate and identification. Journal of Family Business Strategy, 5(3), 289-299.

\section{Introducción}

Una empresa familiar (EF) se puede definir como una empresa gobernada y/o administrada por una coalición dominante controlada por miembros de la misma familia con la intención de transferirla a través de generaciones de la misma (Chua, Chrisman, \& Sharma, 1999). Por tanto, la toma de decisiones estratégicas en las EFs está fuertemente influenciada por la familia y la creación de valor en estas empresas implica obtener tanto resultados económicos como no económicos. Estos resultados no económicos (o no financieros) están básicamente relacionados con la riqueza socioemocional familiar. Algunos ejemplos son el deseo de la familia de mantener el control de la empresa, preservar los lazos y valores familiares para perpetuar la dinastía familiar y su estilo de vida, así como el prestigio y la imagen de la empresa (e.g., Berrone, Cruz, \& Gómez-Mejia, 2012).

El clima familiar, los lazos y las dinámicas familiares son factores determinantes en la compleja relación familia-empresa $\mathrm{y}$ tienen consecuencias en aspectos fundamentales como la gestión de la empresa y su ventaja competitiva. En consecuencia, las diferencias entre las familias propietarias en términos de estructura, valores, emociones y patrones de relación son una fuente importante de heterogeneidad de las EFs en términos de valoración de la riqueza socioemocional y de fijación de objetivos no financieros (ONFs) (Berrone y otros, 2012). Sobre esta base, este trabajo analiza cómo las dinámicas familiares influyen en el grado en que las EFs establecen ONFs, y proponemos que un clima familiar positivo genera un mayor grado de identificación para los miembros de la familia con la empresa y, a su vez, esa identificación lleva a la empresa a adoptar ONFs.

De esta forma, esta investigación tiene como objetivo arrojar luz sobre preguntas tales como: ¿Por qué algunas familias empresarias terminan vendiendo su legado incluso cuando la empresa

${ }^{1}$ Autor de correspondencia: katiuska.cabrera@ulpgc.es 
tiene éxito y las relaciones familiares no son especialmente problemáticas? En otras palabras, ¿cuál es el papel del clima familiar en la motivación de la familia para preservar su riqueza socioemocional? ¿Es suficiente un clima familiar positivo o se necesita algo más para que las familias propietarias preserven y promuevan su riqueza socioemocional? Concretamente, lo que sugerimos es que la identificación familiar con la empresa es la clave para explicar las diferencias entre las EFs en términos de la importancia dada a los ONFs.

\section{El clima familiar y la identificación con la empresa familiar}

Uno de los pocos intentos de medir el funcionamiento de las familias, y específicamente de las familias empresarias, es el estudio de Björnberg y Nicholson (2007) con su Escala de Clima Familiar para medir diversos aspectos del funcionamiento de una familia como son la cohesión emocional, la cohesión cognitiva, la atención intergeneracional y la comunicación abierta que consideran, a su vez, como los componentes de un clima familiar positivo. Así, la cohesión emocional mide la fortaleza del vínculo emocional percibido por los miembros de la familia. Puede considerarse como el "pegamento" emocional entre parientes que es necesario para construir relaciones y evitar la fragmentación. La cohesión cognitiva es una medida del grado de similitud entre las opiniones, valores, intereses y gustos de los miembros de la familia. La atención intergeneracional refleja el grado de participación e interés de la generación mayor en las actividades, necesidades y objetivos de la generación más joven. La forma en que la generación senior se relaciona con los demás establece los parámetros de conducta para toda la familia y actúa como un molde para la estructura de las relaciones dentro de la familia. Finalmente, la comunicación abierta tiene que ver con el nivel de interacción entre los miembros de la familia en lo que respecta a expresar opiniones y tomarse tiempo para discutir temas y preocupaciones.

Asimismo, una de las funciones principales de una familia es proporcionar una identidad a sus miembros. La identificación social es el proceso por el cual los individuos se ven a sí mismos como parte de un grupo de personas que le sirve como marco de referencia para sus valores, metas y estándares de comportamiento (Nahapiet \& Ghoshal, 1998). En este sentido, podríamos afirmar que las relaciones sociales y los lazos de apego entre los miembros del grupo familiar (clima familiar) son determinantes clave en la construcción de la identidad en una familia empresaria (Pearson, Carr, \& Shaw, 2008). Esto es así porque la cohesión, la comunicación y la atención intergeneracional ayudan a la construcción de relaciones, un funcionamiento familiar saludable, la creación de un liderazgo fuerte basado en normas y valores, y la socialización de los miembros de la familia para aceptar esas normas y valores, es decir, para identificarse con ellos. En consecuencia, las familias empresarias con un clima positivo de relaciones probablemente mostrarán un alto grado de integración entre la identidad familiar y la empresarial (Sundaramurthy \& Kreiner, 2008). Por lo tanto, se establece la siguiente hipótesis:

H1. Cuanto más positivo es el clima familiar, mayor es el grado de identificación que los miembros de la familia tienen con la EF.

\section{Identificación con la empresa familiar y objetivos no financieros}

Los lazos familiares y las experiencias compartidas incrementan la identificación con la familia, y esto, a su vez, ayuda a identificarse con un conjunto común de objetivos relacionados con el cumplimiento de las obligaciones familiares y la preservación de la reputación familiar.

Así, por un lado, la identificación con la familia tiene un poderoso efecto sobre la voluntad de sus miembros para preservar el bien 
común. Por lo tanto, los líderes de una EF actuarán como buenos administradores $\mathrm{y}$ tomarán decisiones orientadas a proteger los activos familiares y pasar un negocio más saludable y más fuerte a las generaciones futuras (Le Breton-Miller \& Miller, 2009). Por otro lado, los miembros de la familia que se identifican con la empresa perciben una superposición entre la identidad de la empresa y la suya y, por lo tanto, tratarán de garantizar que el comportamiento de la empresa sea valorado positivamente por personas externas para mantener una imagen positiva de la empresa y de sí mismos (Deephouse \& Jaskiewicz, 2013). En consecuencia, tendrán especial cuidado en tomar decisiones comerciales que puedan proteger la imagen y reputación de la familia, lo que a su vez implica que se esforzarán por un conjunto particular de objetivos principalmente relacionados con metas no financieras 0 socioemocionales. Por tanto:

H2: Cuanto mayor sea el grado de identificación de los miembros de la familia con la empresa, mayor será la importancia de los ONFs en el proceso de toma de decisiones de la EF.

En conjunto, nuestras dos hipótesis sugieren que tener buenas relaciones familiares no significa que las EFs otorguen automáticamente importancia a los ONFs. Solo cuando la identidad empresarial se entrelaza con la identidad familiar, los miembros de la familia experimentarán un sentimiento de propiedad emocional del negocio. Este sentimiento es lo que marca la diferencia en el grado de importancia otorgado a los ONFs.

\section{Metodología}

Dado que en España no existe una base de datos oficial de EFs no cotizadas el equipo de investigación creó esta base de datos al identificar indirectamente a las EFs a partir de la información proporcionada por Informa Dun y Bradstreet. Para ello, se ha considerado que una empresa es familiar si al menos dos personas en el consejo de administración y/o equipo de gestión tienen nombres de pila diferentes y dos apellidos idénticos (es decir, son hermanos); y si algunas (o varias) de las personas que ocupan puestos de alta dirección tienen al menos uno de estos dos apellidos (es decir, él/ella es un miembro de la familia). De esta forma aseguramos que las empresas identificadas sean esencialmente EFs, dado que existirá una influencia familiar real en la toma de decisiones y una transmisión del liderazgo entre generaciones en la familia. Aplicando estos criterios 693 empresas fueron finalmente seleccionadas para este estudio.

La información necesaria para la investigación empírica se obtuvo mediante encuestas estructuradas administradas a miembros de los equipos de alta dirección (EADs) de las empresas seleccionadas. El resultado fue una muestra de $173 \mathrm{EFs}(24,96 \%$ de tasa de respuesta) distribuidas en España, obteniendo 374 cuestionarios válidos. $\mathrm{La}$ encuesta incluyó a más de un informante por empresa, como una forma de reducir el impacto de las percepciones individuales y obtener evaluaciones más objetivas.

El perfil demográfico de las empresas indica que la mayoría de ellas tienen más de 30 años y están en su segunda generación, que pertenecen al sector secundario, seguido por el sector servicios, y tienen entre 50 y 249 empleados. El porcentaje de propiedad familiar es mayoritario y el presidente del consejo, el CEO, o el director general es un miembro de la familia. Por lo tanto, en estas empresas las familias ejercen un alto nivel de control sobre las mismas en términos de propiedad y participación en la gestión.

Para el contraste de hipótesis se utilizó un modelo de ecuaciones estructurales cuyos resultados (véase figura 1) demuestran que (1) el clima familiar positivo es un antecedente directo de la identificación con la EF, validando así la $\mathrm{H} 1$; y que (2) la identificación con la EF es un 
antecedente directo de la importancia que las EFs le dan a los ONFs, aceptándose así la H2. Estos hallazgos indican que la importancia que las EFs le dan a los ONFs puede explicarse directamente por la identificación de los miembros de los equipos directivos con la $\mathrm{EF}$, e indirectamente por el clima familiar positivo a través de esta identificación.

Figura 1. Resultados del modelo propuesto

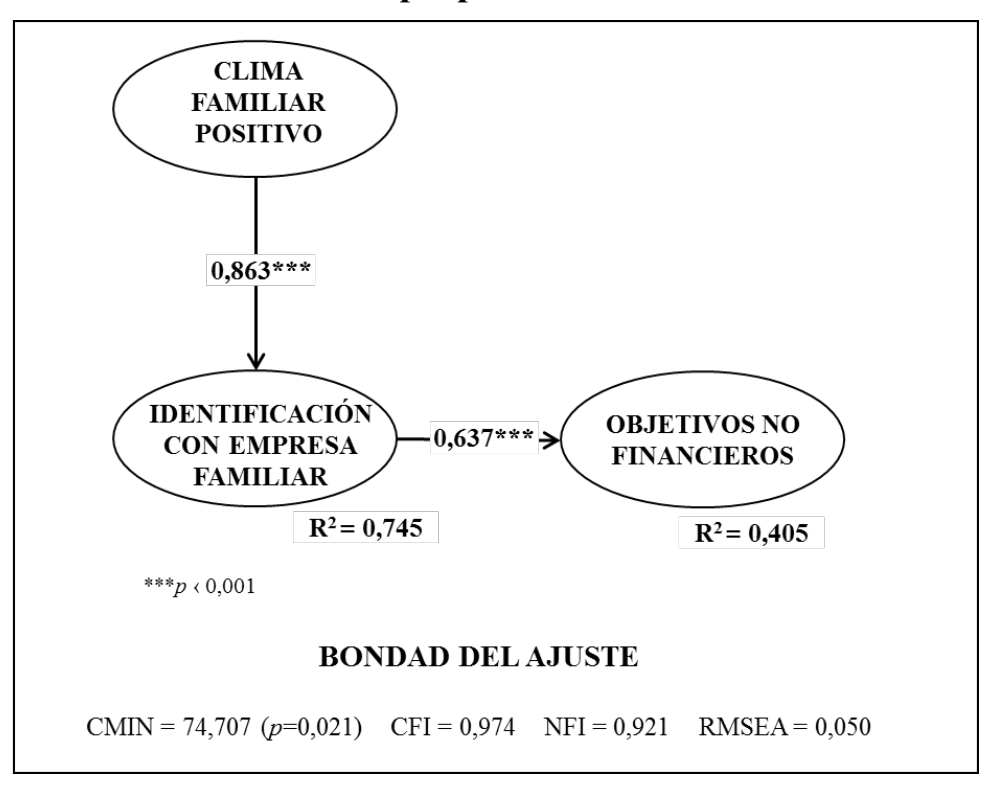

muy similar; el efecto de la identificación con la EF sobre la importancia dada a los ONFs es positivo y significativo en ambos grupos, pero para el grupo 1 dicho efecto es más fuerte; se explica en una proporción considerable la identificación con la EF en ambos grupos pero, en menor medida, la importancia dada a los ONFs, siendo casi el doble en el grupo 1.

\section{Implicaciones}

Esta investigación ha demostrado que el énfasis que las EFs ponen en los ONFs depende del grado de identificación que los miembros de la familia tengan con la empresa, la cual a su vez depende del clima de relaciones dentro de las familias empresarias. Por tanto, se puede concluir que el clima familiar es un factor importante para garantizar la preservación de la riqueza socioemocional de la familia empresaria siempre que se logre generar también un sentido de identificación familiar con la empresa. Cuanto mayor es el grado de presencia familiar en la gestión de la EF, más importante es el papel de la identificación en la relevancia dada a los ONFs.

Por otro lado, se incluyeron tres variables de control en el modelo: el tamaño y la generación de la empresa, y el porcentaje de directivos familiares en los EADs. Esta última variable mostró un efecto positivo y significativo sobre la importancia dada por las empresas a los ONFs. Como consecuencia, se consideró apropiado profundizar en el análisis de la influencia de la composición del EAD. En este sentido, decidimos ajustar el modelo tanto para el grupo de empresas en el que el porcentaje de directivos familiares es igual o superior al 50\% (grupo 1: EAD familiar) y el grupo de empresas en el que dicho porcentaje es inferior al $50 \%$ (grupo 2: EAD no familiar). Los resultados de este modelo estructural multigrupo, que se muestran en la figura 2, indican que: el efecto del clima familiar positivo en la identificación con la EF es positivo y significativo en ambos grupos, además de ser
Desde un punto de vista práctico, nuestros hallazgos resaltan la importancia del gobierno de las familias empresarias. Los órganos $\mathrm{e}$ instrumentos tales como los consejos de familia $\mathrm{y}$ protocolos familiares pueden ser esenciales para garantizar el compromiso de los miembros de la familia con la supervivencia y el desarrollo a largo plazo de la empresa, y para transmitir este compromiso a los equipos de gestión. En este sentido, aunque los miembros de la familia generalmente dominan los EADs en las EFs, la influencia de los directivos no familiares en la toma de decisiones estratégicas también podría ser relevante. Por lo tanto, es importante que experimenten identificación con la empresa y se comprometan a apoyar las decisiones tomadas. Como resultado, la dinámica de las relaciones entre los miembros de los EADs (familiares y no familiares) debe gestionarse adecuadamente, 
dada la influencia significativa que pueden tener aspectos como la cohesión, la comunicación y la confianza en el funcionamiento de estos equipos y en el grado de identificación organizativa de todos sus miembros.

\section{Figura 2. Resultados del modelo multigrupo}

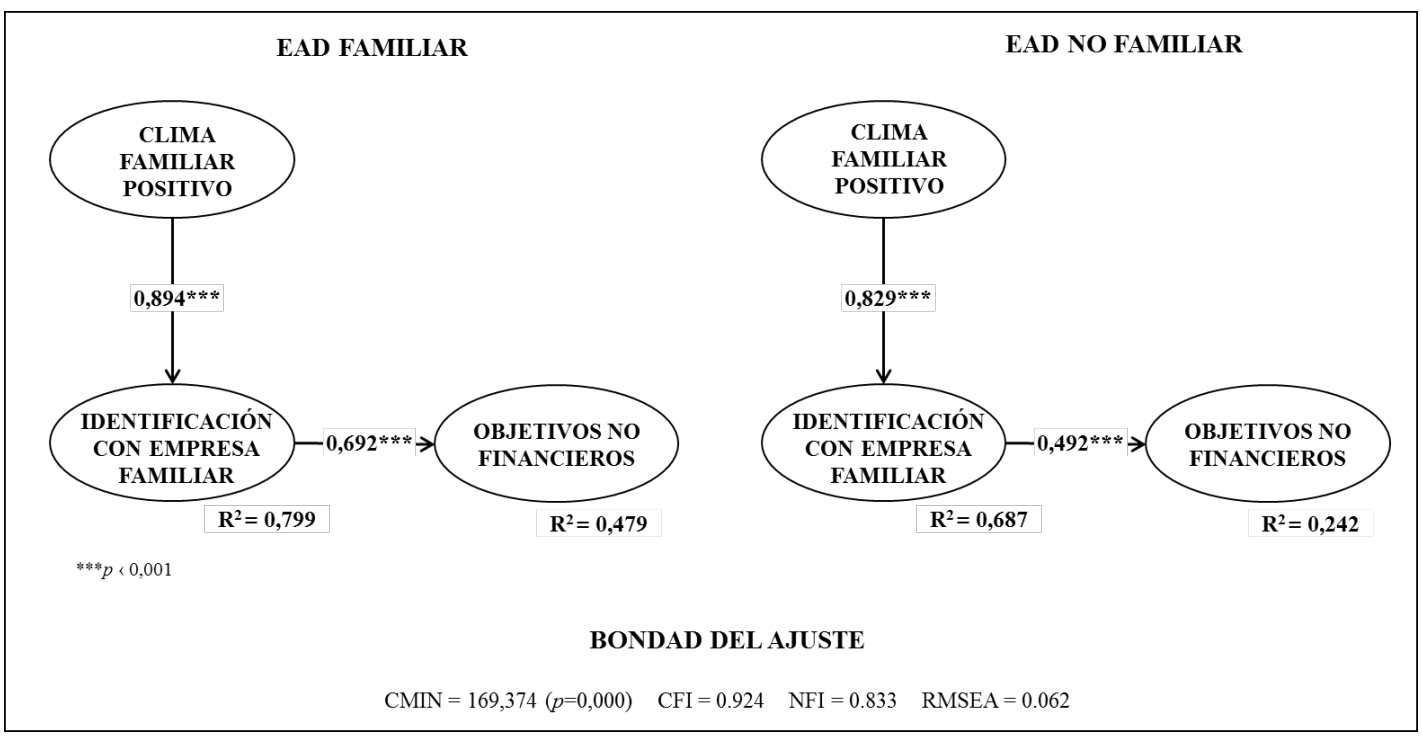

\section{Referencias}

Berrone, P., Cruz, C., \& Gomez-Mejia, L.R. (2012). Socioemotional wealth in family firms: Theoretical dimensions, assessment approaches, and agenda for future research. Family Business Review, 25, 258279

Björnberg, A., \& Nicholson, N. (2007). The family climate scales: Development of a new measure for use in family business research. Family Business Review, 20, 229-246.

Chua, J.H., Chrisman, J.J., \& Sharma, P. (1999). Defining the family business by behavior. Entrepreneurship: Theory and Practice, 23, 19-39.

Deephouse, D.L., \& Jaskiewicz, P. (2013). Do family firms have better reputations than non-family firms? An integration of Socioemotional Wealth and Social
Identity theories. Journal of Management Studies, 50, $337-360$.

Le Breton-Miller, I., \& Miller, D. (2009). Agency vs. stewardship in public family firms: A social embeddedness reconciliation. Entrepreneurship Theory and Practice, 33, 1169-1191.

Nahapiet, J., \& Ghoshal, S. (1998). Social capital, intellectual capital, and the organizational advantage. Academy of Management Review, 23, 242-266.

Pearson, A.W., Carr, J.C., \& Shaw, J.C. (2008). Toward a theory of familiness: A social capital perspective. Entrepreneurship Theory and Practice, 32, 949-969.

Sundaramurthy, C., \& Kreiner, G.E. (2008) Governing by managing identity boundaries: The case of family businesses. Entrepreneurship Theory and Practice, 32, 415-436. 